\title{
Interaction between Periodic System of Rigid Inclusions and Rectilinear Cohesive Cracks in an Isotropic Medium under Transverse Shear
}

\author{
Vagif M. Mirsalimov, Fuad F. Hasanov \\ Azerbaijan Technical University \\ Baku, Azerbaijan \\ E-mail: mir-vagif@mail.ru,hff74@mail.ru
}

\begin{abstract}
We consider a plane problem of fracture mechanics for an isotropic medium with a periodic system of circular holes filled with absolutely rigid inclusions soldered along the contour and weakened by rectilinear cracks with interfacial bonds at the end zones collinear to the abscissa and ordinate axes of unequal length under transverse shear. The problem on equilibrium of isotropic composite medium with cohesive cracks is reduced to the solution of the system of nonlinear singular integro-differential equations with Cauchy type kernel. The tangential forces at the end zones of the cracks are found from the solution of this system of equations. The crack propagation condition is stated with regard to ultimate stretching of the material bonds.
\end{abstract}

Keywords: isotropic medium; periodic system of circular holes; cohesive forces; rigid inclusions; transverse shear; prefracture zone; cracks with interfacial bonds

\section{Introduction}

At present, technical means, in the form of perforated elements are used in many fields of engineering. Therefore, development of strength analysis methods of perforated elements of machines and constructions is of great value. Investigation of these problems is important in connection with development of power engineering, chemical industry and other branches of engineering and also with wide use of periodic structure materials.

By investigating the stress distribution in shear of the plane perpendicular to fibers (inclusions) orientation, one can get a good notion on typical stress distributions in the microstructure of reinforced materials. The solution of this problem opens new opportunities for mechanical properties forecasting of composite materials on given initial characteristics, for constituent components and in the form of microstructure. At the design stage of new machines and structures it is necessary to take into account the cases when, in components of the machines and/or 
structures, there may appear cracks. A large amount of literature has been devoted to these problems (see review of the papers in $[1,2]$ ). In a great majority of papers, the authors have considered only the Criffith's cracks, i.e. the cracks with not interacting edges. In structurally-inhomogeneous materials, in availability of violated structure zones near the crack, a considerable part of the crack is drawn into the failure process. In this case the fracture zone may be considered as some end-zone adjoining to the crack with a material with partially violated interparticle couplings. Among the investigations of the last years we can note the papers [311].

\section{Formulation of the Problem}

Begin with an isotropic medium weakened by a system of circular holes of radii $\lambda(\lambda<1)$ and the centers at the points

$$
P_{m}=m \omega \quad(m=0, \pm 1, \pm 2, \ldots), \omega=2
$$

The circular holes of the medium are filled with absolutely rigid inclusions soldered along the contour. In [12] the investigations were limited to the consideration of Griffith's cracks. In the present study, the isotropic medium is weakened with two periodic systems of rectilinear cohesive cracks collinear to the abscissa and ordinate axes of unequal length (Fig. 1). The crack faces outside of the end zones are free from external loads. The plane under consideration is subjected to transverse shear by the forces $\tau_{x y}^{\infty}$. It is required to determine the stress strain state in the isotropic medium according to boundary conditions on non-availability of elastic displacements, along the contour of circular holes and the external loads on the faces of periodic system of cracks outside end zones.

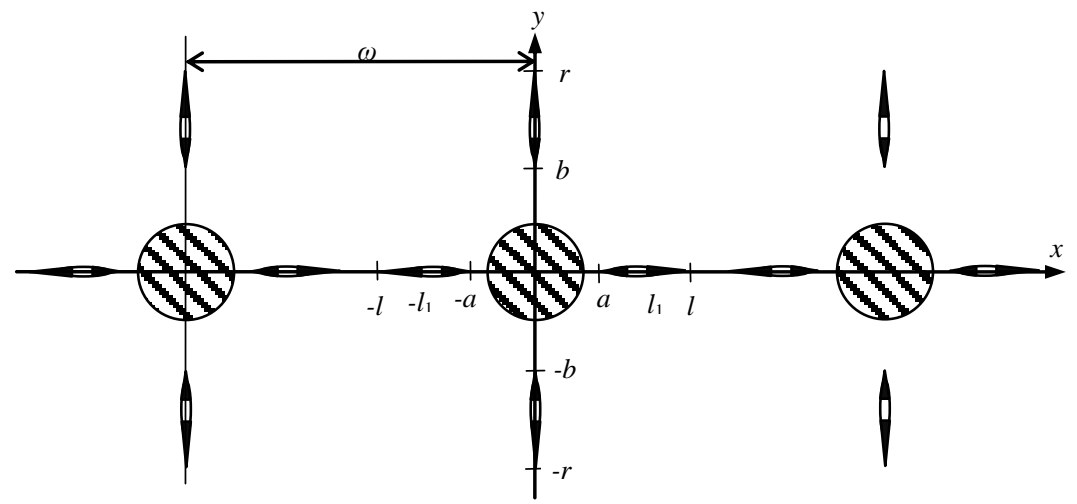

Figure 1

Calculation scheme of the problem on interaction of rigid inclusions and cohesive cracks 
As the external load $\tau_{x y}^{\infty}$ increases, there will arise concluding prefracture zones on the continuation of rectilinear cracks. The model bridged cracks at the end zones are used [13-21]. The crack's end zones are modeled by the areas with weakened interparticle bonds in the medium material. Interaction of faces of these zones are modeled by introducing bonds with the given deformation diagram between the prefracture zone faces. The physical nature of such bonds and the sizes of the prefracture zones depend on the form of the material.

When the external load $\tau_{x y}^{\infty}$ acts on the composite body, in bonds connecting the faces of end prefracture zones, there arise tangential forces $q_{x}(x)$ and $q_{y}(y)$, respectively. These stresses are not known beforehand and should be defined.

Due to the symmetry of boundary conditions and geometry of the domain $D$ occupied with the material, the stresses are periodic functions with the main period $\omega$.

The boundary conditions of the problem have the form

$u+i v=0$ on the contours of circular holes

And on the crack faces

$\sigma_{y}-i \tau_{x y}=0$ collinear to the abscissa axis

$\sigma_{x}-i \tau_{x y}=0$ collinear to the ordinate axis

For the faces of end prefrature zones

$\sigma_{y}-i \tau_{x y}=-i q_{x}(x)$ collinear to the abscissa axis

$\sigma_{x}-i \tau_{x y}=-i q_{y}(y)$ collinear to the ordinate axis

The basic relations of the stated problem should be complemented with the relationships connecting the shear of prefracture zone faces and forces in the bonds. Without loss of generality, we will represent these equations in the form

$$
\begin{aligned}
& u^{+}(x, 0)-u^{-}(x, 0)=C\left(x, q_{x}(x)\right) q_{x}(x) \\
& v^{+}(0, y)-v^{-}(0, y)=C\left(y, q_{y}(y)\right) q_{y}(y)
\end{aligned}
$$

where the functions $C\left(x, q_{x}(x)\right)$ and $C\left(y, q_{y}(y)\right)$ are effective compliances of the bonds, $\left(u^{+}-u^{-}\right)$is the shear of the faces of end prefracture zones collinear to abscissa axes; $\left(v^{+}-v^{-}\right)$is the shear of the faces of end prefracture zones collinear to ordinate axis.

To determine the ultimate quantity of the external load under which the crack propagation occurs, the problem statement should be complemented with crack 
propagation condition (criterion). In place of such a condition we take [22] a deformational fracture criterion (critical shear of prefracture zone faces) on the faces of end prefrature zones

$u^{+}-u^{-}=\delta_{\mathrm{II}} \quad$ collinear to the abscissa axis

$v^{+}-v^{-}=\delta_{\text {II }} \quad$ collinear to the ordinate axis

where $\delta_{\mathrm{II}}$ is the crack resistance characteristics of the medium material.

\section{The Method of the Boundary-Value Problem Solution}

In order to solve the problem in a natural way we combine the method worked out by solving the periodic elastic problem [2] with the method [23] for constructing in the explicit form the Kolosov-Muskhelishvili potentials, corresponding to unknown tangential displacements along cracks with the end zones. We represent the stresses and displacements [24] by the Kolosov-Muskheleshvili potentials $\Phi(z)$ and $\Psi(z)$

$$
\begin{aligned}
& \sigma_{x}+\sigma_{y}=4 \operatorname{Re} \Phi(z), \Phi(z)=\varphi^{\prime}(z), \Psi(z)=\psi^{\prime}(z) \quad(z=x+i y) \\
& \sigma_{y}-\sigma_{x}+2 i \tau_{x y}=2\left[\bar{z} \Phi^{\prime}(z)+\Psi(z)\right] \\
& 2 \mu(u+i v)=\kappa \varphi(z)-z \overline{\Phi(z)}-\overline{\psi(z)}
\end{aligned}
$$

where $\kappa=3-4 v$ for plane strain, $\kappa=(3-v) /(1+v)$ for plane stress state; $\mu$ and $v$ is a shear modulus and the Poisson's ratio, respectively. Based around formulas (6) and boundary conditions on the contours of circular holes and on the surfaces of cracks with end zones, the problem is reduced to definition of two analytic functions $\Phi(z)$ and $\Psi(z)$ in domain $D$ from the boundary conditions ( $t$ and $t_{1}$ are the affices of the points of the crack surfaces with end zones collinear to abscissa and ordinate axes, respectively)

$$
\begin{aligned}
& \varepsilon \overline{\Phi(\tau)}+\Phi(\tau)-\left[\bar{\tau} \Phi^{\prime}(\tau)+\Psi(\tau)\right] e^{2 i \theta}=0 \\
& \Phi(t)+\overline{\Phi(t)}+t \overline{\Phi^{\prime}(t)}+\overline{\Psi(t)}=f_{x}(t) \\
& \Phi\left(t_{1}\right)+\overline{\Phi\left(t_{1}\right)}+t_{1} \overline{\Phi^{\prime}\left(t_{1}\right)}+\overline{\Psi\left(t_{1}\right)}=f_{y}\left(t_{1}\right) \\
& \text { where } \tau=\lambda e^{i \theta}+m \omega \quad(m=0, \pm 1, \pm 2, \ldots)
\end{aligned}
$$


$f_{x}(t)= \begin{cases}0 & \text { on free faces of cracks collinear to the abscissa } O x \text { axis } \\ -i q_{x}(t) & \text { on the faces of crack' s end zones }\end{cases}$ $f_{y}\left(t_{1}\right)=\left\{\begin{array}{l}0 \quad \text { on free faces of cracks collinear to the ordinate } O y \text { axis } \\ -i q_{y}\left(t_{1}\right) \quad \text { on the faces of crack' s end zones }\end{array}\right.$

The problem statement simultaneously covers the cases of rigid inclusions $(\varepsilon=-\kappa)$ and free holes $(\varepsilon=1)$. We look for the solution of boundary value problem (7)-(8) in the form

$$
\begin{aligned}
& \Phi(z)=\Phi_{1}(z)+\Phi_{2}(z)+\Phi_{3}(z), \Psi(z)=\Psi_{1}(z)+\Psi_{2}(z)+\Psi_{3}(z) \\
& \Phi_{1}(z)=\frac{1}{2 \omega} \int_{L_{1}} g(t) \cot \frac{\pi}{\omega}(t-z) d t \\
& \Psi_{1}(z)=-\frac{\pi z}{2 \omega^{2}} \int_{L_{1}} g(t) \sin ^{-2} \frac{\pi}{\omega}(t-z) d t \\
& \Phi_{2}(z)=\frac{i}{2 \omega} \int_{L_{2}} g_{1}\left(t_{1}\right) \cot \frac{\pi}{\omega}\left(i t_{1}-z\right) d t_{1} \\
& \Psi_{2}(z)=-\frac{i}{2 \omega} \int_{L_{2}} g_{1}\left(t_{1}\right)\left[2 \cot \frac{\pi}{\omega}\left(i t_{1}-z\right)+\frac{\pi}{\omega}\left(2 t_{1}+i z\right) \sin ^{2} \frac{\pi}{\omega}\left(i t_{1}-z\right)\right] d t_{1} \\
& \Phi_{3}(z)=i \tau_{x y}^{\infty}+i \sum_{k=0}^{\infty} \alpha_{2 k+2} \frac{\lambda^{2 k+2} \rho^{(2 k)}(z)}{(2 k+1) !} \\
& \Phi_{3}(z)=i \tau_{x y}^{\infty}+i \sum_{k=0}^{\infty} \beta_{2 k+2} \frac{\lambda^{2 k+2} \rho^{(2 k)}(z)}{(2 k+1) !}-i \sum_{k=0}^{\infty} \alpha_{2 k+2} \frac{\lambda^{2 k+2} S^{(2 k+1)}(z)}{(2 k+1) !}
\end{aligned}
$$

where $\rho(z)=\left(\frac{\pi}{\omega}\right)^{2} \sin ^{-2}\left(\frac{\pi}{\omega} z\right)-\frac{1}{3}\left(\frac{\pi}{\omega}\right)^{2} ; \quad S(z)=\sum_{m}^{\prime}\left[\frac{P_{m}}{\left(z-P_{m}\right)^{2}}-\frac{2 z}{P_{m}}-\frac{1}{P_{m}}\right]$, the prime of the sum sign indicates that the subscript $m=0$ is excluded from the summation; the integrals in (10) are taken on the line $L_{1}=[-l,-a] \cup[a, l]$, $L_{2}=[-r,-b] \cup[b, r] ; g(t)$ and $g\left(t_{1}\right)$ are the desired functions characterizing the shear of crack faces with end zones

$$
\begin{aligned}
& g(x)=-\frac{2 \mu i}{1+\kappa} \frac{d}{d x}\left[u^{+}(x, 0)-u^{-}(x, 0)\right] \text { on } L_{1} \\
& g_{1}(y)=\frac{2 \mu}{1+\kappa} \frac{d}{d y}\left[v^{+}(0, y)-v^{-}(0, y)\right] \text { on } L_{2}
\end{aligned}
$$


To relations (9)-(12) we should add additional conditions following from the physical sense of the problem

$$
\begin{aligned}
& \int_{-l}^{-a} g(t) d t=0, \int_{a}^{l} g(t) d t=0 \\
& \int_{-r}^{-b} g_{1}\left(t_{1}\right) d t_{1}=0, \quad \int_{b}^{r} g_{1}\left(t_{1}\right) d t_{1}=0
\end{aligned}
$$

Refer to the dependences to which the coefficients of equations (9)-(11) should satisfy. From the anti-symmetry conditions for the coordinate axes we find

$$
\operatorname{Im} \alpha_{k}=0, \quad \operatorname{Im} \beta_{k}=0 \quad(k=1,2, \ldots)
$$

From the condition of constancy of the principal vector of all forces acting on the arch, connecting two congruent points in $D$ it follows that

$$
\alpha_{0}=\frac{\pi^{2}}{24} \beta_{2} \lambda^{2}
$$

It is easy to see that the functions (9)-(11) under condition (13) determine the class of problems with periodic distribution of stresses.

The unknown functions $g(x), g_{1}(y)$ and the constants $\alpha_{2 k}$ and $\beta_{2 k}$ should be defined from boundary conditions (7) and (8). Subject to the periodicity conditions, the system of boundary conditions (7) is replaced by one functional equation, for example on the contour $\tau=\lambda e^{i \theta}$, the system of conditions (8) by the boundary conditions on $L_{1}$ and $L_{2}$.

To compose the equations with respect to the coefficients $\alpha_{2 k}$ and $\beta_{2 k}$ of the functions $\Phi_{3}(z)$ and $\Psi_{3}(z)$ we represent boundary conditions (7) in the form

$$
\begin{aligned}
& \varepsilon \overline{\Phi_{3}(\tau)}+\Phi_{3}(\tau)-\left[\bar{\tau} \Phi_{3}^{\prime}(\tau)+\Psi_{3}(\tau)\right] e^{2 i \theta}=f_{1}(\theta)+i f_{2}(\theta)+\varphi_{1}(\theta)+i \varphi_{2}(\theta) \\
& f_{1}(\theta)+i f_{2}(\theta)=-\varepsilon \overline{\Phi_{1}(\tau)}-\Phi_{1}(\tau)+\left[\bar{\tau} \Phi_{1}^{\prime}(\tau)+\Psi_{1}(\tau)\right] e^{2 i \theta} \\
& \varphi_{1}(\theta)+i \varphi_{2}(\theta)=-\varepsilon \overline{\Phi_{2}(\tau)}-\Phi_{2}(\tau)+\left[\bar{\tau} \Phi_{2}^{\prime}(\tau)+\Psi_{2}(\tau)\right] e^{2 i \theta}
\end{aligned}
$$

For the functions $f_{1}(\theta)+i f_{2}(\theta)$ and $\varphi_{1}(\theta)+i \varphi_{2}(\theta)$ we will assume that they expand on $|\tau|=\lambda$ in Fourier series. By anti-symmetry, these series have the form

$$
\begin{aligned}
& f_{1}(\theta)+i f_{2}(\theta)=\sum_{k=-\infty}^{\infty} A_{2 k} e^{2 i k \theta}, \quad \operatorname{Re} A_{2 k}=0 \\
& A_{2 k}=\frac{1}{2 \pi} \int_{0}^{2 \pi}\left[f_{1}(\theta)+i f_{2}(\theta)\right] e^{-2 i k \theta} d \theta \quad(k=0, \pm 1, \pm 2, \ldots)
\end{aligned}
$$




$$
\begin{aligned}
& \varphi_{1}(\theta)+i \varphi_{2}(\theta)=\sum_{k=-\infty}^{\infty} B_{2 k} e^{2 i k \theta}, \operatorname{Re} B_{2 k}=0 \\
& B_{2 k}=\frac{1}{2 \pi} \int_{0}^{2 \pi}\left[\varphi_{1}(\theta)+i \varphi_{2}(\theta)\right] e^{-2 i k \theta} d \theta \quad(k=0, \pm 1, \pm 2, \ldots)
\end{aligned}
$$

Substituting to these series relation (12) and changing the integration order, after calculation of integrals by means of residue we find

$$
\begin{aligned}
& A_{k}=-\frac{1}{2 \omega} \int_{L_{1}} g(t) f_{2 k}(t) d t \\
& f_{0}(t)=(1+\varepsilon) \gamma(t), \quad \gamma(t)=\cot \frac{\pi}{\omega} t \\
& f_{2}(t)=-\frac{\lambda^{2}}{2} \gamma^{(2)}(t) \\
& f_{2 k}(t)=-\frac{(2 k-1)}{(2 k) !} \lambda^{2 k} \gamma^{(2 k)}(t)+\frac{1}{(2 k-3) !} \lambda^{2 k-2} \gamma^{(2 k-2)}(t) \quad(k=2,3, \ldots) \\
& f_{-2 k}(t)=-\frac{\varepsilon}{(2 k) !} \lambda^{2 k} \gamma^{(2 k)}(t) \quad(k=1,2, \ldots) \\
& B_{2 k}=-\frac{i}{2 \omega} \int_{L_{2}} g_{1}\left(t_{1}\right) \varphi_{2 k}\left(i t_{1}\right) d t_{1} \\
& \varphi_{0}\left(i t_{1}\right)=\frac{1}{2}(1+\varepsilon)\left[\delta\left(i t_{1}\right)-\overline{\delta\left(i t_{1}\right)}\right], \quad \delta\left(i t_{1}\right)=\cot \frac{\pi}{\omega}\left(i t_{1}\right) \\
& \varphi_{2}\left(i t_{1}\right)=-\frac{\lambda^{2}}{2} \delta^{(2)}\left(i t_{1}\right)+2\left[\delta\left(i t_{1}\right)-i t_{1} \delta^{\prime}\left(i t_{1}\right)\right] \\
& \varphi_{2 k}\left(i t_{1}\right)=\frac{(1-2 k) \lambda^{2 k}}{(2 k) !} \delta^{(2 k)}\left(i t_{1}\right)+\frac{2 \lambda^{2 k-2}}{(2 k-2) !}\left[k \delta^{(2 k-2)}\left(i t_{1}\right)-i t_{1} \delta^{(2 k-1)}\left(i t_{1}\right)\right] \quad(k=1,2, \ldots) \\
& \varphi_{-2 k}\left(i t_{1}\right)=-\frac{\varepsilon \lambda^{2}}{(2 k) !} \delta^{(2 k)}\left(i t_{1}\right) \quad(k=1,2, \ldots)
\end{aligned}
$$

Substituting to the left side of boundary condition (14) instead of $\Phi_{3}(\tau), \overline{\Phi_{3}(\tau)}$, $\Phi_{3}^{\prime}(\tau)$ and $\Psi_{3}(\tau)$ their expansion in Laurent series in the vicinity of $z=0$, and to the right side of (14) the Fourier series (16) and comparing the coefficients at the same degrees of $e^{i \theta}$ we get two infinite system of algebraic equations with respect to the coefficients $\alpha_{2 k}$ and $\beta_{2 k}$ 


$$
\begin{aligned}
& i \alpha_{2 j+2}=\sum_{k=0}^{\infty} i a_{j, k} \alpha_{2 k+2}+b_{j} \quad(j=0,1,2, \ldots) \\
& a_{j, k}=\frac{2 j+1}{\varepsilon} \gamma_{j, k} \lambda^{2 k+2 j+2} \\
& \gamma_{0,0}=\frac{3}{8} g_{2} \lambda^{2}+\varepsilon \sum_{k=1}^{\infty} \frac{(2 j+1) g_{i+1}^{2} \lambda^{4 i+2}}{2^{4 i+4}} \\
& \gamma_{j, k}=\frac{(2 j+2 k+2) ! g_{j+k+1}}{(2 j+1) !(2 k+1) ! 2^{2 i+2 k+2}}+\frac{(2 j+2 k+4) ! g_{j+k+2} \lambda^{2}}{(2 j+2) !(2 k+2) ! 2^{2 i+2 k+4}}+ \\
& +\frac{g_{j+1} g_{k+1} \lambda^{2}}{2^{2 i+2 k+4}}\left[1+\frac{(1+\varepsilon)^{2} K_{2} \lambda^{2}}{1-(1+\varepsilon) K_{2} \lambda^{2}}\right]+ \\
& +\varepsilon \sum_{i=0}^{\infty} \frac{(2 j+2 i+1) !(2 k+2 i+1) ! g_{j+i+1} g_{k+i+1} \lambda^{4 i+2}}{(2 j+1) !(2 k+1) !(2 i+1) !(2 i) ! 2^{2 j+2 k+4 i+4}} \quad(j, k=1,2, \ldots) \\
& \varepsilon b_{0}=A_{2}^{\prime}-\sum_{k=0}^{\infty} \frac{g_{k+2} \lambda^{2 k+4}}{2^{2 k+4}} A_{-2 k-2}^{\prime} \\
& \varepsilon b_{j}=A_{2 j+2}^{\prime}-\frac{(2 j+1) g_{j+1} \lambda^{2 j+4}}{\left(1-(1+\varepsilon) K_{2} \lambda^{2}\right) 2^{2 j+2}} A_{0}^{\prime}-\sum_{k=0}^{\infty} \frac{(2 j+2 k+3) ! g_{j+k+2} \lambda^{2 j+2 k+4}}{(2 j) !(2 k+3) ! 2^{2 j+2 k+4}} A_{-2 k-2}^{\prime} \\
& g_{j}=2 \sum_{m=1}^{\infty} \frac{1}{A_{0}^{2 j}}=A_{0}+B_{0}-2 i \tau_{x y}^{\infty}, A_{2}^{\prime}=A_{2}+B_{2}+i \tau_{x y}^{\infty} \\
& A_{2 k}^{\prime}=A_{2 k}+B_{2 k}(k= \pm 1, \pm 2, \ldots) \\
& (j=1,2, \ldots), K_{2}=\frac{\pi^{2}}{24}
\end{aligned}
$$

The coefficients $\beta_{2 k}$ are determined by the relation

$$
\begin{aligned}
& i \beta_{2}=\frac{1}{1-(1+\varepsilon) K_{2} \lambda^{2}}\left[-A_{0}^{\prime}+(1+\varepsilon) \sum_{k=1}^{\infty} \frac{g_{k+1} \lambda^{2 k+2} i \alpha_{2 k+2}}{2^{2 k+2}}\right] \\
& i \beta_{2 j+4}=i(2 j+3) \alpha_{2 j+2}+\varepsilon \sum_{k=0}^{\infty} \frac{(2 j+2 k+3) ! g_{j+k+2} \lambda^{2 j+2 k+2}}{(2 j+2) !(2 k+1) ! 2^{2 j+2 k+4}} i \alpha_{2 k+2}-A_{-2 j-2}^{\prime}
\end{aligned}
$$

Requiring that functions (9)-(11) should satisfy the boundary condition on the faces of the crack with end zone $L_{1}$, we get a singular integral equation with respect to $g(x)$

$$
\frac{1}{\omega} \int_{L_{1}} g(t) \cot \frac{\pi}{\omega}(t-z) d t+H(x)=f_{x}(x)
$$




$$
\begin{aligned}
& H(x)=\Phi_{S}(x)+\overline{\Phi_{S}(x)}+x \overline{\Phi_{S}^{\prime}(x)}+\overline{\Psi_{S}(x)} \\
& \Phi_{S}(z)=\Phi_{2}(z)+\Phi_{3}(z), \Psi_{S}(z)=\Psi_{2}(z)+\Psi_{3}(z)
\end{aligned}
$$

Similarly, satisfying the boundary condition on the line $L_{2}$ after some transformations we get one more singular integral equation with respect to the desired function $g_{1}(y)$

$$
\begin{aligned}
& -\frac{\pi}{\omega^{2}} \int_{L_{1}} g_{1}(t)\left[(t-y) \operatorname{sh}^{-2} \frac{\pi}{\omega}(t-y)\right] d t+N(y)=f_{y}(y) \\
& N(y)=\Phi_{0}(i y)+\overline{\Phi_{0}(i y)}+i y \overline{\Phi_{0}^{\prime}(i y)}+\overline{\Psi_{0}(i y)} \\
& \Phi_{0}(z)=\Phi_{1}(z)+\Phi_{3}(z), \Psi_{0}(z)=\Psi_{1}(z)+\Psi_{3}(z)
\end{aligned}
$$

Systems (17) and (18) together with singular integral equations (19) and (20) are the main resolving equations of the problem and allow to define the functions $g(x), g_{1}(y)$ and the coefficients $\alpha_{2 k}, \beta_{2 k}$.

\section{Method of Numerical Solution and Analysis}

Using the expansion of the functions $\cot \frac{\pi}{\omega} z, \operatorname{sh}^{-2} \frac{\pi}{\omega} z$ in the main strip of periods, and also using the substitution of variables, after some transformations we will reduce the singular integral equations to the standard form. Using the quadrature formulas [23, 25], we reduce main resolving equations (17), (18), (19), (20) to the totality of two infinite system of linear algebraic equation and to two finite algebraic systems with respect to approximate values $p_{k}^{0}=g\left(\eta_{k}\right)$ $(k=1,2, \ldots, M), R_{v}^{0}=g_{1}\left(\eta_{v}\right) \quad(v=1,2, \ldots, M)$ of the desired functions at the nodal points

$$
\begin{aligned}
& \sum_{v=1}^{M} A_{m v} p_{v}^{0}+\frac{1}{2} H_{*}\left(\eta_{m}\right)=f_{x}\left(\eta_{m}\right) \quad(m=1,2, \ldots, M-1) \\
& \sum_{v=1}^{M} \frac{p_{v}^{0}}{\sqrt{1 / 2\left(1-\lambda_{1}^{2}\right)\left(\tau_{v}+1\right)+\lambda_{1}^{2}}}=0 \\
& \sum_{v=1}^{M} B_{m v} R_{v}^{0}+\frac{1}{2} N_{*}\left(\eta_{m}\right)=f_{y}\left(\eta_{m}\right) \quad(m=1,2, \ldots, M-1) \\
& \sum_{v=1}^{M} \frac{R_{v}^{0}}{\sqrt{1 / 2\left(1-\lambda_{2}^{2}\right)\left(\tau_{v}+1\right)+\lambda_{2}^{2}}}=0
\end{aligned}
$$


Here $A_{m v}=\frac{1}{2 M}\left[\frac{1}{\sin \theta_{m}} \cot \frac{\theta_{m}+(-1)^{|m-v|} \theta_{v}}{2}+B\left(\eta_{m}, \tau_{v}\right)\right]$,

$$
\begin{aligned}
& \theta_{m}=\frac{2 m-1}{2 M} \pi \quad(m=1,2, \ldots, M), \tau_{m}=\cos \theta_{m}, \eta_{m}=\tau_{m}, \lambda_{1}=a / l \\
& B(\eta, \tau)=-\frac{1-\lambda_{1}^{2}}{2} \sum_{j=0}^{\infty} g_{j+1}\left(\frac{l}{2}\right)^{2 j+2} u_{0}^{j} A_{j} \\
& A_{j}=(2 j+1)+\frac{(2 j+1)(2 j)(2 j-1)}{1 \cdot 2 \cdot 3}\left(\frac{u}{u_{0}}\right)+\ldots+ \\
& +\frac{(2 j+1)(2 j)(2 j-1) . .[(2 j+1)-(2 j+1-1)]}{1 \cdot 2 \cdot \ldots \cdot(2 j+1)}\left(\frac{u}{u_{0}}\right)^{j} \\
& u=\frac{1-\lambda_{1}^{2}}{2}(\tau+1)+\lambda_{1}^{2}, u_{0}=\frac{1-\lambda_{1}^{2}}{2}(\eta+1)+\lambda_{1}^{2} \\
& B_{m v}=\frac{1}{2 M}\left[\frac{1}{\sin \theta_{m}} \cot \frac{\theta_{m}+(-1)^{|m-v|} \theta_{v}}{2}+B_{*}\left(\eta_{m}, \tau_{v}\right)\right] \\
& B_{*}(\eta, \tau)=-\frac{1-\lambda_{2}^{2}}{2} \sum_{j=0}^{\infty}(-1)^{j}(2 j+1) g_{j+1}\left(\frac{r}{2}\right)^{2 j+2} u_{1}^{j} A_{j}^{\prime} \\
& A_{j}^{\prime}=(2 j+1)+\frac{(2 j+1)(2 j)(2 j-1)}{1 \cdot 2 \cdot 3}\left(\frac{u_{1}}{u_{2}}\right)+\ldots+\left(\frac{u_{1}}{u_{2}}\right)^{j} \\
& 2
\end{aligned}
$$

The right side of the obtained systems contains the unknown stresses $q_{x}\left(\eta_{m}\right)$ and $q_{y}\left(\eta_{m}\right)$ at the nodal points, belonging to prefracture zones. Using the obtained solution, we represent of equation (12) in the form

$$
\begin{aligned}
& g(x)=-\frac{2 \mu i}{1+\kappa} \frac{d}{d x}\left[C\left(x, q_{x}(x)\right) q_{x}(x)\right] \\
& g_{1}(y)=\frac{2 \mu}{1+\kappa} \frac{d}{d y}\left[C\left(y, q_{y}(y)\right) q_{y}(y)\right]
\end{aligned}
$$

These equations help to determine the traction at the bonds of the end zones of cracks. For constructing missing equations conditions (23) should be fulfilled at the nodal points. We use the finite differences method. And as a result we get two more systems from $M_{1}$ and $M_{2}$ equations in order to determine approximate values 
$q_{x}\left(\eta_{m_{1}}\right) \quad\left(m_{1}=1,2, \ldots, M_{1}\right)$ and $q_{y}\left(\eta_{m_{2}}\right) \quad\left(m_{2}=1,2, \ldots, M_{2}\right)$. Since, in the perforated body the stresses are finite, the solution of singular integral equations should be sought in the class of universally bounded functions. Consequently, to system (21)-(22) we should add the conditions of stress bounded environment at the crack vertices

$$
\begin{aligned}
& \sum_{k=1}^{M}(-1)^{k+M} p_{k}^{0} \tan \frac{\theta_{k}}{2}=0, \sum_{k=1}^{M}(-1)^{k} p_{k}^{0} \cot \frac{\theta_{k}}{2}=0 \\
& \sum_{v=1}^{M}(-1)^{v+M} R_{v}^{0} \tan \frac{\theta_{v}}{2}=0, \sum_{v=1}^{M}(-1)^{v} R_{v}^{0} \cot \frac{\theta_{v}}{2}=0
\end{aligned}
$$

The obtained systems of equations (17), (18), (21)-(24) completely define the solution of the problem. For numerical realization of the stated method the calculations were performed. Each of the infinite system of equations were reduced to five equations. In numerical calculations $M=30$ that corresponds to portioning of integration interval into 30 Chebyshev nodes. Since the sizes of the end zones are unknown, the resolving algebraic system of equations (17)-(18); (21)-(24) of the problem is nonlinear even for linear-elastic bonds. For its solution the sequential approximations method is used [25]. The essence of this method is solving the algebraic system for some definite values of end zones sizes with respect to the remaining unknowns. The remaining unknowns enter the resolving system linearly. The accepted values of the end zone sizes and the appropriate values of the remaining unknowns will not satisfy, generally speaking, the stress bounded conditions at the crack vertices. Therefore, choosing the values of end zone sizes, we will repeat calculations until the stress bounded conditions of (24) will be satisfied with the given accuracy. In the case of nonlinear law of deformation of bonds, for determination of tangential forces at the end prefracture zones, the iteration algorithm similar to the method of elastic solutions [26] was used. It is assumed that the law of deformation of inter-particle bonds at the end prefracture zone is linear for $\left(u^{+}-u^{-}\right) \leq u_{*}$ and $\left(v^{+}-v^{-}\right) \leq v_{*}$. The first step of iterative calculation process is to solve the systems of equations for linear-elastic bonds. The next iterations are fulfilled only in the case when the inequality $\left(u^{+}-u^{-}\right)>u_{*}$ or $\left(v^{+}-v^{-}\right)>v_{*}$ holds on the part of the end prefracture zone. For such iterations, the system of equations at each approximation is solved for quasilinear bonds with effective compliance changing along the end zone forces and dependent on the quantity of forces in bonds that was calculated at the preceding step. Calculation of effective compliance is conducted as in definition of the secant modulus in the method of variables of elasticity parameters [27]. The sequential approximations process finishes when the forces along the end zone, obtained on two sequential iterations do not differ at all. The nonlinear part of the curve of bonds deformation was approximated by the nonlinear dependence whose ascending section corresponded to deformation of bonds $\left(0<\left(u^{+}-u^{-}\right) \leq u_{*}\right)$ with their maximal traction of bonds. For $\left(u^{+}-u^{-}\right)>u_{*}$ the 
deformation law was described by nonlinear dependence determined by the points $\left(u_{*}, \tau_{*}\right)$ and $\left(\delta_{c}, \tau_{c}\right)$, for $\tau_{c} \geq \tau_{*}$ an ascending linear dependence hold (linear strengthening corresponding to elastico-plastic deformation of bonds).

In order to determine the limit equilibrium state of the medium under which the crack propagates, we use condition (5). Using the obtained solution, by the conditions defining the ultimate external load we find the followings:

$C\left(d, q_{y}(d)\right) q_{y}(d)=\delta_{\text {II }}$

$C\left(d^{*}, q_{x}\left(d^{*}\right)\right) q_{x}\left(d^{*}\right)=\delta_{\text {II }}$

Here $d$ and $d^{*}$ are the coordinates of the points at the foundation of the end prefracture zones, respectively. The length of the end zone, tractions in bonds and shear of opposite faces of end prefracture zone from the loading parameter $\tau_{x y}^{\infty}$ were found as a result of numerical calculation. The dependence of relative length of the end prefracture zone $l_{*}=\left(l-l_{1}\right) / \lambda$ on dimensionless value of the external load $\tau_{x y}^{\infty} / \tau_{*}$ for different values of the radius of holes (curves 1-4): $1-\lambda=0,2 ; 2$ $-x=0,3 ; 3-\lambda=0,4 ; 4-\lambda=0,5$ are represented in Fig. 2 .

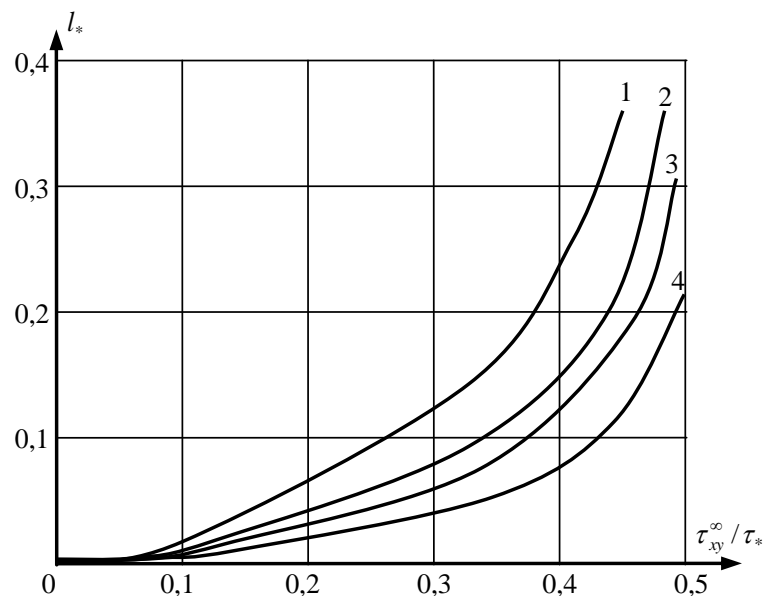

Figure 2

Dependence of relative length of the end prefracture zone $l_{*}=\left(l-l_{1}\right) / \lambda$ on dimensionless value of the external load $\tau_{x y}^{\infty} / \tau_{*}$ for different values of the radius of holes (curves 1-4): $1-\lambda=0,2 ; 2-$

$$
\lambda=0,3 ; 3-\lambda=0,4 ; 4-\lambda=0,5
$$

The dependence of tractions in the bonds $q_{x} / \tau_{x y}^{\infty}$ in relative size of $l_{*}$ for different values of the radius of holes; $\lambda=0,2 \div 0,5$ (curves 1-4) are cited in Fig. 3 .

The joint solution of the algebraic system and condition (25) enables (for the given characteristic of material crack resistance) to define the critical quantity of 
the external load, the sizes of end zones of the cracks for the limit-equilibrium state under which the crack propagates.

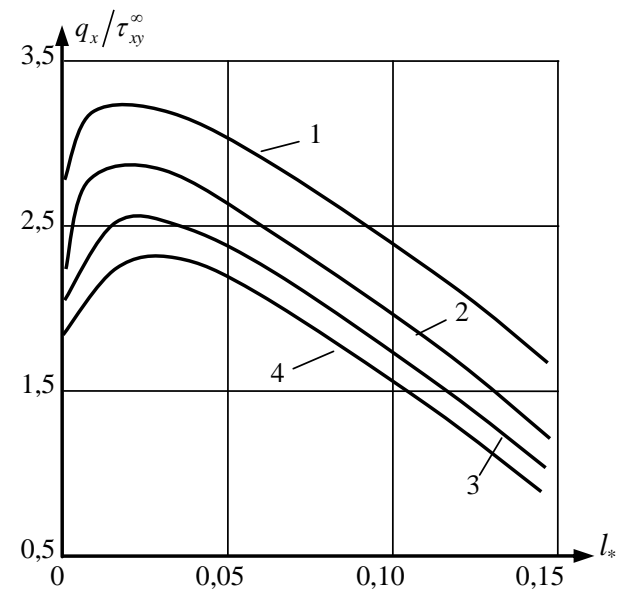

Figure 3

Traction distribution in bonds $q_{x} / \tau_{x y}^{\infty}$ from the relative size $l_{*}$ of the end zone for different values of the radius of the holes $\lambda=0,2 \div 0,5$ (curves $1-4$ )

On the base of the obtained numerical results, the graphs of dependence of critical load $\tau^{*}=\tau_{x y}^{\infty} / \tau_{*}$ for the distance $a_{*}=a-\lambda$ for the both ends of cracks collinear to the abscissa axis (curve 1 corresponds to the left end) for $\lambda=0.3$ are structured in Fig. 4.

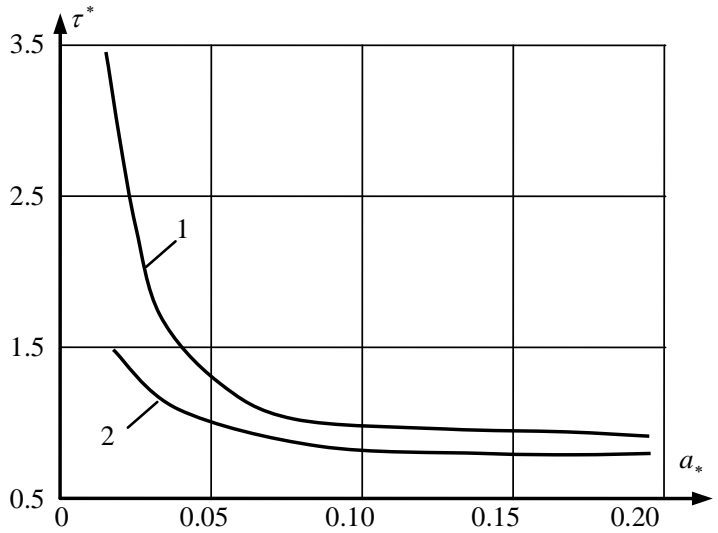

Figure 4

Dependence of critical load $\tau^{*}=\tau_{x y}^{\infty} / \tau_{x}$ on the distance $a_{*}=a-\lambda$ for the both ends of the cracks, collinear to the abscissa axis (curve 1 corresponds to the left end) for $\lambda=0,3$ 
The dependence of critical load $\tau^{* a}$ when the crack length $l_{*}=l-a$ changes is represented in Fig. 5. For $\lambda=0.3, a_{*}=0.05$

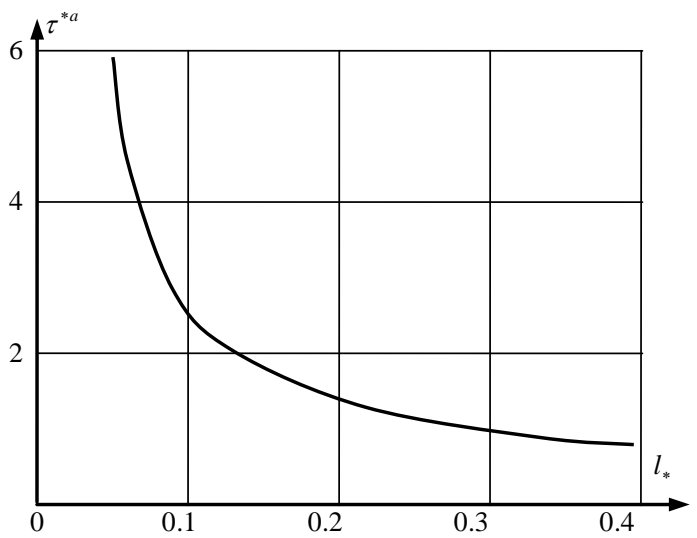

Figure 5

Dependence of critical load $\tau^{* a}$ when the crack's length $l_{*}=l-a$ changes, for $\lambda=0.3, a_{*}=0.05$

\section{Conclusions}

The analysis of the limit equilibrium state of a body with periodic system of rigid inclusions and rectilinear cracks, with interfacial bonds at the end zones under transverse shear, is reduced to a simultaneous parametric investigation of resolving algebraic system (17), (18), (21), (22)-(24) and deformation criterion of fracture (25) under different laws of deformations of interparticle bonds of the material, elastic constants and geometric characteristics of a perforated body. The traction in bonds and shear of the crack surfaces are determined directly from the solution of the obtained algebraic systems.

The model of the cohesive crack allows us to investigate the main regularities of traction distributions in bonds under different deformation laws; to analyze the limit equilibrium of the crack with end prefracture zone, with regard to deformational condition of fracture; to estimate the critical external load and material's crack resistance. The obtained relations enable to investigate the limit equilibrium state of the medium with a periodic system of circular holes filled with absolutely rigid inclusions soldered along the contour and weakened with bridged rectilinear cracks at the end zones collinear to abscissa and ordinate axes of unequal length under transverse shear.

\section{References}

[1] Savruk M. P.: 'Fracture Mechanics and Strength of Materials. Vol. 2: Stress Intensity Factors in Cracked Bodies'. Kiev, Naukova Dumka (1988) (in Russian) 
[2] Mirsalimov V. M.: 'Fracture of Elasto - and Elastoplastic Bodies with Cracks'. Baku, Science, 1984 (in Russian)

[3] Elices M., Guinea G. V., Gómez J., Planas J.: 'The Cohesive Zone Model: Advantages, Limitations and Challenges', Engineering Fracture Mechanics, Vol. 69, Issue 2, pp. 137-163, 2002

[4] Yang Q., Cox B.: 'Cohesive Models for Damage Evolution in Laminated Composites International' Journal of Fracture, Vol. 133, Issue 2, pp 107137,2005

[5] Dong C. Y.: 'The Integral Equation Formulations of an Infinite Elastic Medium Containing Inclusions, Cracks and Rigid Lines', Engineering Fracture Mechanics, Vol. 75, Issue 13, pp. 3952-3965, 2008

[6] Ghannad M., Zamani Nejad M.: 'Elastic Analysis of Heterogeneous Thick Cylinders Subjected to Internal or External Pressure Using Shear Deformation Theory', Acta Polytechnica Hungarica, Vol. 9, No. 6, pp. 117136, 2012

[7] Pasternak Ia. M., Vasil'ev K. V., Sulym H. T.: 'Antiplane Deformation by Concentrated Factors of Bounded Bodies with Cracks and Rigid Inclusions', Journal of Mathematical Sciences, Vol. 190, Issue 5, pp. 710-724, 2013

[8] Xiao J. H., Xu Y. L., Zhang F. C.: 'Interaction between Periodic Cracks and Periodic Rigid-Line Inclusions in Piezoelectric Materials', Acta Mechanica, Vol. 224, pp. 777-787, 2013

[9] Hasanov F. F.: 'Fracture of a Body Weakened with Periodic System of Circular Orifices at Transverse Shear', Materials. Technologies, Tools, Vol. 18, No. 1. pp. 17-23. 2013

[10] Caimmi F., Pavan A.: 'A Numerical Study of Crack-Fibre Interaction at Varying Fibre Orientation', Engineering Fracture Mechanics, Vol. 101, pp. 129-139, 2013

[11] Kun Zhou, Hsin Jen Hoh, Xu Wang, Leon M. Keer, John H. L. Pang, Bin Song, Q. Jane Wang: 'A Review of Recent Works on Inclusions', Mechanics of Materials, Vol. 60, pp. 144-158, 2013

[12] Mirsalimov V. M.: 'Interaction between a Periodic System of Elastic Inclusions and Rectilinear Cracks in an Isotropic Medium', Journal of Applied Mechanics and Technical Physics January-February, Vol. 19, Issue 1, pp. 136-145, 1978

[13] The special issue: Cohesive Models, Eng. Fract. Mech., Vol. 70, No. 14, pp. 1741-1987, 2003

[14] Mirsalimov V. M: 'Cracks with Bonding between the Lips in Bushings of Friction Couples’, Materials Science, Vol. 42, Issue 2, pp 200-209, 2006 
[15] Mir-Salim-zade M. V.: 'Crack Initiation in a Stiffened Plate', Journal of Applied Mechanics and Technical Physics, Vol. 48, Issue 4, pp. 562-570, 2007

[16] Mirsalimov V. M.: 'The Solution of a Problem in Contact Fracture Mechanics on the Nucleation and Development of a Bridged Crack in the Hub of a Friction Pair', Journal of Applied Mathematics and Mechanics, Vol. 71, Issue 1, pp. 120-136, 2007

[17] Mirsalimov V. M.: 'Nucleation of Cracks in a Perforated Fuel Cell', Journal of Applied Mechanics and Technical Physics, Vol. 48, Issue 5, pp. 723-733, 2007

[18] Mirsalimov V. M., B. E. Rustamov: 'Interaction of Prefracture Zones and Crack-Visible Cavity in a Burning Solid with Mixed Boundary Conditions', Acta Mechanica, Vol. 223, Issue 3, pp. 627-643, 2012

[19] Zolgharnein E., Mirsalimov V. M.: 'Nucleation of a Crack under Inner Compression of Cylindrical Bodies', Acta Polytechnica Hungarica, Vol. 9, No. 2, pp. 169-183, 2012

[20] Mirsalimov V. M., Zolgharnein E.: 'Cracks with Interfacial Bonds in the Hub of a Friction Pair', Meccanica, Vol. 47, No. 7, pp. 1591-1600, 2012

[21] Mirsalimov V. M., Rustamov B. E.: 'Effect of Damages on Crack-Visible of the Cavity Opening Displacement in Burning Solid Fuel', Inter. J. of Damage Mechanics, Vol. 21, No. 3, pp. 373-390, 2012

[22] Panasyuk V. V.: 'Deformation Criteria in Fracture Mechanics', Materials Science, Vol. 22, No. 1, pp. 7-17, 1986

[23] Panasyuk V. V., Savruk M. P., Datsyshyn A. P.: 'A General Method of Solution of Two-Dimensional Problems in the Theory of Cracks', Eng. Fract. Mech., No. 2, pp. 481-497, 1977

[24] Muskhelishvili N. I.: 'Some Basic Problems of Mathematical Theory Elasticity’ Amsterdam, Kluwer, 1977

[25] Mirsalimov V. M.: 'Non-One-Dimensional Elastoplastic Problems' Nauka, Moscow, 1987 (in Russian)

[26] Il'yushin A. A.: 'Plasticity' Moscow and Leningrad, Gostexhizd, 1948 (in Russian)

[27] Birger I. A.: 'The Design of Structures Allowings for Plasticity and Creep', Izv. Akad. Nauk SSSR, Mekhanika, No. 2, pp. 113-119, 1965 